\title{
Eventos catastróficos associados ao tratamento da comunicação interatrial tipo ostium secundum: razões para não se subestimar este tipo de cardiopatia congênita
}

Catastrophic events associated to the surgical treatment of ostium secundum atrial septal defects. Reasons for not underestimating this type of congenital cardiopathy

Paulo Roberto B. EVORA, Paulo José de Freitas RIBEIRO, Walter V.V. VICENTE, Antonio C. MENARDI, Alfredo J. RODRIGUES, Celso L. REIS, Fernanda VIARO

\section{Resumo}

Objetivo: $O$ presente trabalho clínico foi motivado pela frustrante experiência de quatro pacientes operados para o tratamento cirúrgico da comunicação interatrial tipo ostium secundum (CIA-II), que vieram a falecer em condições extremamente dramáticas.

Método: Estudo retrospectivo embasado em dados de prontuários. As pesquisas bibliográficas incluíram: tromboembolismo paradoxal (cerebral, pulmonar ou mesentérico), malformações vasculares do sistema nervoso central e conexões anômalas das veias cavas. Estas pesquisas da literatura foram embasadas em possíveis eventos, inesperados e catastróficos, que levaram quatro pacientes ao óbito.

Resultados: Os quatros pacientes, todos do sexo feminino, foram submetidos a atriosseptorrafia com tempo de parada cardíaca isquêmica inferior a 20 minutos, em circulação extracorpórea. As causas de óbito foram: isquemia intestinal não-oclusiva, ruptura de aneurisma cerebral de artéria comunicante anterior, cor pulmonale com hipertensão arterial e tromboembolismo e um provável tromboembolismo cerebral em uma criança que precisou ser reoperada pela drenagem da veia cava inferior em átrio esquerdo.

Conclusão: A lição final deste trabalho é: "Não subestime a comunicação interatrial em cirurgia cardíaca!’.

Descritores: Defeitos do septo interatrial, cirurgia, complicações. Cardiopatias congênitas, cirurgia, complicações. Circulação extracorpórea.

\section{Abstract}

Objective: The present article was motivated by the frustrating experiences with four patients who underwent surgical treatment of ostium secundum atrial septal defect (ASD-II) and who died in extremely dramatic circumstances.

Method: This is a retrospective study based on clinical data. The bibliographical researche included: paradoxical thromboembolism (cerebral, lung or mesenteric), central nervous system vascular malformations and anomalous vena

Trabalho realizado no Hospital do Coração de Ribeirão Preto Fundação Waldemar B. Pessoa. CECORP - Centro Especializado do Coração e Pulmão de Ribeirão Preto. Disciplina de Cirurgia Torácica e Cardiovascular Faculdade de Medicina de Ribeirão Preto - USP

Endereço para correspondência: Paulo Roberto B. Évora. Rua Rui Barbosa, 367, apto. 15. Ribeirão Preto, SP, Brasil. CEP14015-120. E-mail:prbevora@keynet.com.br 
cava connections. This research was based on possible events, unexpected and catastrophic, that could have directly caused the patients' deaths.

Results: All patients were female, the operations were performed under cardiopulmonary bypasses with less than 20 minutes of aortic cross-clamping. The causes of death were: non-occlusive intestinal ischemia, rupture of a cerebral aneurysm of the anterior communicating artery, cor pulmonale associated with pulmonary arterial hypertension and thromboembolism and probable cerebral thromboembolism in a child that needed to be reoperated to correct anomalous

\section{INTRODUÇÃO}

A comunicação interatrial tipo ostium secundum (CIAII) é uma das cardiopatias congênitas mais freqüentes. Embora seja compatível até com idades superiores a 70 anos, está muito bem estabelecido que pacientes portadores de CIA têm uma menor expectativa de vida e uma maior morbimortalidade quando comparados com uma população normal. Por este motivo, recomenda-se o seu tratamento cirúrgico na idade pré-escolar, mesmo na ausência de sintomas, ou quando diagnosticada da idade adulta [1-3].

O presente trabalho foi motivado pela frustrante experiência de quatro pacientes operados de CIA-II, que vieram a falecer em condições extremamente dramáticas. Todos as pacientes eram do sexo feminino e foram submetidas a atriosseptorrafia com tempo de parada cardíaca isquêmica inferior a 20 minutos, em circulação extracorpórea. Artigo de GUNAY [4], resume, já em seu título, toda a idéia deste texto: "Não subestime a comunicação interatrial em cirurgia cardíaca".

\section{RELATODOS CASOS}

Caso 1. Paciente branca, 22 anos, com história de palpitações e dispnéia aos médios e pequenos esforços havia 4 anos. Durante a sua primeira gravidez os sintomas acentuaram-se intensamente, constatando-se a presença de um sopro sistólico no foco pulmonar e bloqueio incompleto do ramo direito detectado pelo eletrocardiograma.

Em maio de 1982, após parto cesárea, foi submetida a cateterismo cardíaco que revelou a presença de uma CIA-II com "shunt" 2,5/1. Aguardou-se o período de amamentação, mas a cirurgia cardíaca, entretanto, teve que ser adiada por nova gravidez, que foi levada a termo com outra operação cesariana.

Realizou-se uma atriosseptorrafia direta, com auxílio de circulação extracorpórea (CEC) e proteção miocárdica com cardioplegia cristalóide. O tempo de operação foi de 80 minutos, com tempos de 30 minutos de circulação extracorpórea e 15 minutos de anóxia.

No terceiro dia, após a alta da UTI, a paciente apresentou súbito episódio de hipotensão arterial e taquicardia com inferior vena cava drainage into the left atrium.

Conclusion: Even being technically easy to treat, death due to atrial septal defects in extremely dramatic circumstances is possible, so "Do not underestimate the atrial septal in congenital heart surgery".

Descriptors: Heart septal defects, atrial, surgery, complications. Heart defects, congenital, surgery, complications. Extracorporeal circulation.

sinais de intensa vasodilatação periférica. Tentou-se a reversão do quadro hemodinâmico por meio de expansão volumétrica e dobutamina endovenosa. Após dois dias de instabilidade cardiocirculatória, já hemodinamicamente controlada, a paciente passou a relatar cólicas periumbilicais e diarréia com fezes escuras e fétidas. $\mathrm{O}$ hemograma tornouse francamente infeccioso, com aumento dos níveis de bilirrubinas e transaminases (TGP e TGO). No oitavo dia do pós-operatório, correspondente ao quinto dia após o início da dor abdominal, a paciente tornou-se dispnéica, com persistência da dor em cólica abdominal. Indicou-se laparotomia exploradora que, ao exame macroscópico e à palpação, não evidenciou trombose mesentérica, mas sofrimento difuso com necrose e isquemia em regiões aleatórias. Realizou-se ampla ressecção intestinal, deixandose apenas cerca de $60 \mathrm{~cm}$ de intestino delgado e metade dos colos descendente e sigmóide (Figura 1). A paciente permaneceu em alimentação parenteral, vindo a falecer após 6 dias da operação abdominal em quadro de falência de múltiplos órgãos por septicemia. Ao exame anátomopatológico, a ausência de trombose em áreas não-inflamadas, associada com áreas de intensa necrose sem um padrão anatômico, bem como a dominância de lesões da mucosa, sugeriu fortemente tratar-se de isquemia intestinal nãooclusiva.
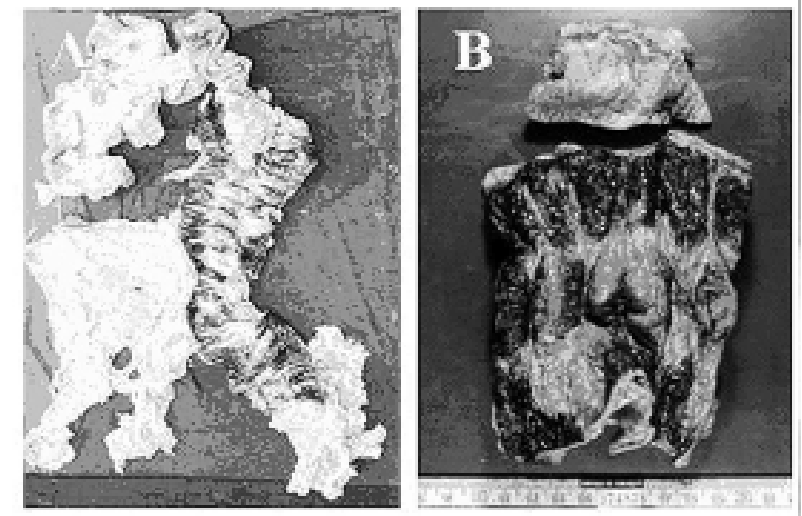

Fig. 1 - Caso 1: A-Alças intestinais ressecadas; B - Detalhe de alça ressecada com a pigmentação devida à provável melanose coli. 
Caso 2. Paciente branca, 31 anos, com palpitações e dispnéia a médios e grandes esforços. Apresentava todos os comemorativos de uma CIA: sopro sistólico suave em foco pulmonar, bloqueio incompleto do ramo direito e hiperfluxo pulmonar à radiografia de tórax e CIA-II claramente identificada pelo ecodopplercardiograma e cateterismo cardíaco. A correção cirúrgica, com auxílio de CEC, foi realizada em março de 1989. Realizou-se atriosseptorrafia direta da CIA-II, que media $4 \mathrm{~cm} \times 2 \mathrm{~cm}$, sem nenhum incidente cirúrgico. A operação durou 135 minutos, o tempo de CEC foi de 35 minutos para um tempo de anóxia miocárdica de 20 minutos. Cerca de duas horas após, já na UTI, apresentou pico hipertensivo, com pressão arterial média superior a $200 \mathrm{mmHg}$, seguido de breve convulsão e acentuada bradicardia. Seguiu-se midríase paralítica e completa arreflexia. A hipótese diagnóstica levantada foi de hemorragia intracraniana, confirmada por punção liquórica. $\mathrm{O}$ neurocirurgião realizou carotidoangiografia à beira do leito, que revelou a ruptura de um grande aneurisma da artéria comunicante anterior (Figura 2). Nenhuma medida terapêutica surtiu efeito, a paciente permaneceu em coma profundo, arreflexia, midríase paralítica, vindo a falecer no terceiro dia do pós-operatório devido à dissociação eletromecânica do coração.
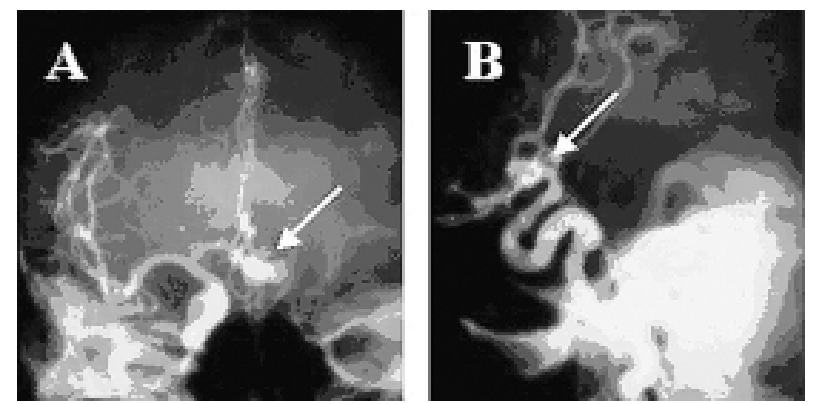

Fig. 2 - Caso 2: A - carotidoangiografia (ântero-posterior) e; B carotidoangiografia (perfil). As setas indicam o aneurisma da artéria comunicante anterior.

Caso 3. Paciente do sexo feminino, atendida pela primeira vez aos 2 anos e 11 meses, com história de descompensação respiratória e broncoespasmo.

Aos 6 anos de idade voltou a apresentar crises de bronquites com freqüência crescente. Indicou-se a operação com base em ecocardiogramas que confirmavam o diagnóstico de CIA-II, não se julgando necessário o cateterismo cardíaco.

A operação foi realizada em agosto de 1992, com auxílio de CEC. O achado cirúrgico mostrou CIA de localização mais alta do que a posição usual da CIA-II (tipo seio venoso?), átrio direito tenso, com grande dificuldade de canulação da veia cava inferior. As veias pulmonares direitas drenavam todas no átrio esquerdo, sendo as superiores posicionadas superiormente e as veias inferiores conectadas mais à esquerda. Realizou-se atriosseptorrafia direta, em dois planos com polipropileno 4-0. Durante a CEC, ocorreu grande dificuldade do retorno venoso para o oxigenador, e o anestesiologista observou que a face da criança estava edemaciada e cianótica. A operação, diante destes fatos, foi realizada tão rápida quanto possível, com um tempo de anóxia miocárdica de 15 minutos e de CEC de 20 minutos. Saiu de CEC em ritmo sinusal espontâneo com boas condições hemodinâmicas. Após a saída de CEC, a criança apresentava pressão de átrio esquerdo normal, cianose e sinais de broncoespasmo. A cianose não se reverteu e, já na UTI, os valores da pressão venosa central apresentavam-se extremamente elevados. Diante deste dado e das ocorrências intra-operatórias, posicionou-se um cateter em veia cava inferior medindo-se pressão de $10 \mathrm{cmH}_{2} \mathrm{O}$. Hipotizou-se uma obstrução de veia cava superior, optando-se por posterior revisão, uma vez que a criança encontrava-se hemodinamicamente bem. Após 10 dias da operação, ainda com cianose mais notada em tronco e membros superiores, apresentou quadro de acidente vascular isquêmico, com hemiplegia direita, recuperada com auxílio de fisioterapia.

Decidiu-se pelo cateterismo cardíaco, o qual revelou: estenose discreta de veia cava superior, septo interatrial íntegro
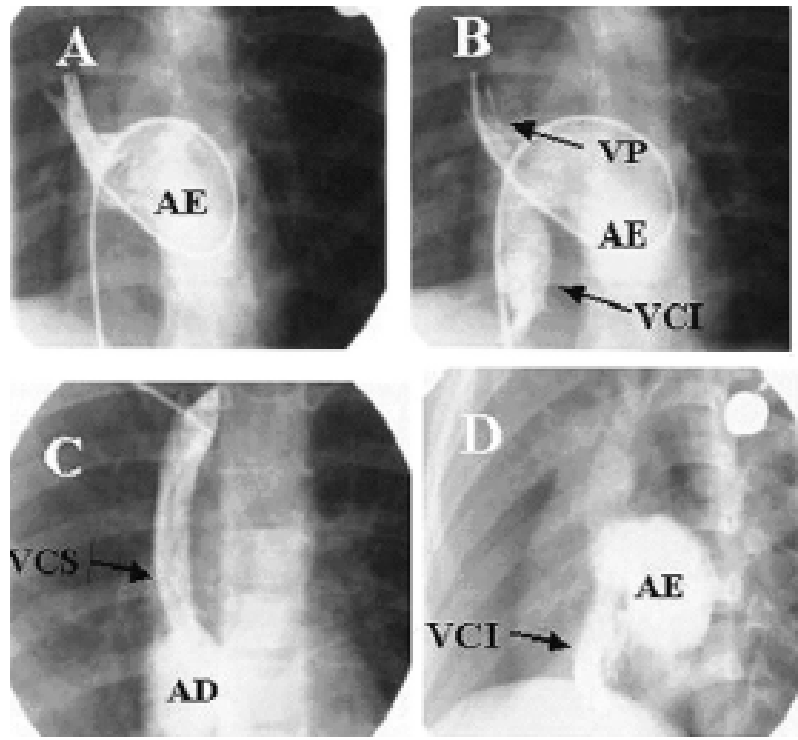

Fig. 3 - Caso 3: A e B - Cateter introduzido pela veia femoral com a extremidade na veia pulmonar superior direita (VP) contrastando nesta fase o átrio esquerdo. A angiografia demonstra drenagem tanto desta veia como da veia cava inferior no átrio esquerdo (AE); C - Injeção de contraste na veia cava superior (VCS) com drenagem normal no átrio direito (AD); D - Incidência oblíqua anterior esquerda com a drenagem da veia cava inferior (VCI) no átrio esquerdo (AE). 
e veia cava inferior conectada ao átrio esquerdo (Figura 3).

Programou-se uma segunda operação cardíaca para reconectar-se a veia cava inferior ao átrio direito. Pela atriotomia direita abriu-se a CIA, ampliou-se o átrio direito inferiormente com auxílio de retalho de pericárdio bovino, reconectando-se a veia cava inferior e, suturando-se novamente a CIA. A evolução no pós-operatório imediato foi muito boa, com desaparecimento da cianose, com a criança voltando a ser hiperativa, que era sua característica. $\mathrm{Na}$ tarde do quinto dia do pós-operatório, correndo no corredor da enfermaria, apresentou morte súbita em assistolia e grande midríase bilateral. $\mathrm{O}$ fato ocorreu diante de um médico da equipe cirúrgica que, de imediato, iniciou massagem cardíaca e ventilação pulmonar. A monitorização do ritmo cardíaco mostrou absoluta falta de atividade miocárdica, sem nenhuma resposta a consideráveis doses de adrenalina endovenosa. Este quadro não se modificou em nada durante cerca de duas horas de manobras de ressuscitação. A família não permitiu a realização de necropsia, ficando como prováveis causas da morte um tromboembolismo do sistema nervoso central (o mais provável) ou embolia "a cavaleiro" do tronco da artéria pulmonar.

Caso 4. Paciente branca, 26 anos, atendida em julho de 1993 com história de cansaço aos grandes esforços que evoluiu para médios esforços no período de um ano. Com eletrocardiograma mostrando sobrecarga de ventrículo direito, radiografia de tórax mostrando hiperfluxo pulmonar e ecodopplercardiograma evidenciando CIA-II com hipertensão pulmonar (pressão estimada entre 90 e 100 $\mathrm{mmHg}$ ), indicou-se o tratamento cirúrgico.

Em agosto de 1993, realizou-se atriosseptorrafia em dois planos, sem a utilização de cardioplegia. O tempo de operação foi de duas horas para um tempo de CEC de 30 minutos e um tempo de anóxia miocárdica de 15 minutos. Sem nenhuma intercorrência pós-operatória, tendo alta após quatro dias.

A paciente manteve seguimento clínico regular e, em junho de 1994, após gestação normal deu à luz um filho saudável por meio de parto cesárea. Passou a referir discreta dispnéia, sem sinais de insuficiência cardíaca e saturação de oxigênio sempre superior a $94 \%$ pela oximetria de pulso. Ecodopplercardiograma realizado quatro anos após a operação, em 1997, não revelou defeitos residuais do septo interatrial e a pressão arterial pulmonar estimada reduziu-se, acentuadamente, para $53 \mathrm{mmHg}$. Em março de 1999, encontrava-se assintomática, mas, em dezembro daquele mesmo ano, retornou com queixa de dispnéia aos grandes esforços e piora progressiva. Encontrava-se acianótica, a ausculta pulmonar revelava uma segunda bulha pulmonar nitidamente hiperfonética e a saturação do sangue arterial, medida pela oximetria de pulso, era de $89 \%$ com valores que atingiam até $86 \%$ quando submetida a esforço isométrico. Diante deste quadro preocupante, desencadeou-se uma série de investigações. A radiografia de tórax revelou aumento de cavidades cardíacas direitas, grande abaulamento da artéria pulmonar e de seus ramos hilares com evidência de diminuição da circulação pulmonar periférica $\mathrm{O}$ eletrocardiograma revelou grande sobrecarga de cavidades direitas com onda P "pulmonale". O ecocardiografista constatou cor pulmonale grave, considerando a possibilidade de transplante cardiopulmonar. As provas de função pulmonar mostraram-se normais quanto à presença de distúrbios obstrutivos e/ou restritivos, saturação de $90 \%$ e $\mathrm{PO}_{2}$ arterial de $62 \mathrm{mmHg}$. A cintilografia de inalação e perfusão pulmonar revelou déficits perfusionais no lobo médio do pulmão direito, com alta probabilidade de tromboembolismo pulmonar. A tomografia computadorizada e angioressonância pulmonar (Figura 4) confirmaram a hipótese de embolia pulmonar. Investigação da coagulação mostrou ausência de anticoagulante lúpico, atividade da proteína $C$ reativa, dosagem da antitrombina III, proteína $S$ (antígeno) total e livre, anticorpos anticardiolipina todos
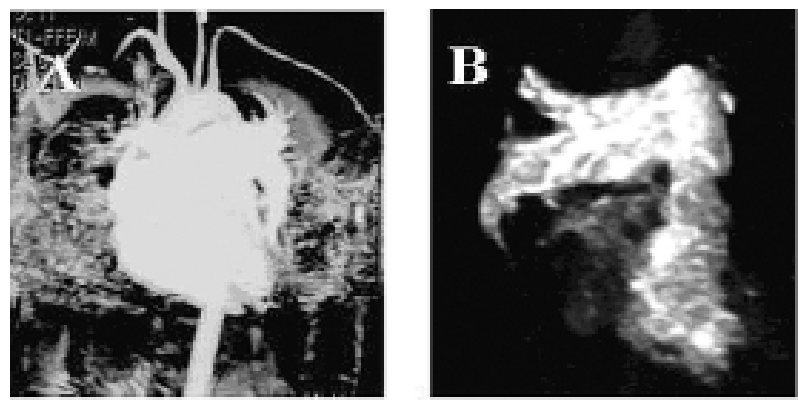

Fig. 4 - Caso 4: Angiorressonância. A-Mostra a artéria pulmonar com o dobro do diâmetro da aorta; B - Ramo esquerdo da artéria pulmonar com falhas de enchimento sugestivas de trombos organizados.

dentro dos parâmetros normais para os métodos utilizados.

Diante desse grave quadro, as opções de conduta foram consideradas: a) Tratamento clínico com vasodilatadores pulmonares (nifedipina?, inibidor da ECA quinapril?) e terapia domiciliar com oxigênio em futuro próximo; b) Transplante cardiopulmonar; c) Transplante pulmonar bilateral; d) Transplante pulmonar unilateral e) Tromboendarterectomia pulmonar. Após várias considerações, levando-se em conta a experiência internacional e a vivência do grupo cirúrgico com a tromboendarterectomia pulmonar, optou-se pela realização deste procedimento.

A operação foi realizada em fevereiro de 2000 , utilizandose $\mathrm{CEC}$, hipotermia a $23^{\circ} \mathrm{C}$ e hipofluxo com períodos curtos de parada circulatória. O rigoroso planejamento baseou-se no protocolo da Universidade de San Diego. Os achados 
cirúrgicos mostraram grande aumento das câmaras cardíacas direitas e dilatação do tronco da artéria pulmonar (cerca do dobro da aorta). Como achado inesperado, verificou-se a presença de uma pequena CIA (cerca de 1,5 x 1,5 cm), próxima à veia cava inferior, a qual foi suturada. Ao se proceder a arteriotomia pulmonar, já foi posta em dúvida a possível melhora da hipertensão pulmonar com alívio do coração direito. Os trombos não eram compatíveis com as imagens obtidas pela tomografia e angioressonância do tórax. A tromboendarterectomia foi bastante convincente, incluindo até alguns ramos terciários da artéria pulmonar esquerda. Não se encontrou trombo no ramo direito, que era dilatado e aterosclerótico. A operação foi realizada sem pinçamento aórtico com tempo de CEC de 220 minutos utilizados para a tromboendarterectomia, tempo de resfriamento e tempo de reaquecimento.

O pós-operatório foi catastrófico. A paciente desenvolveu níveis de pressão pulmonar de até $180 \mathrm{mmHg}$, não se conseguiu diminuir a oferta de oxigênio através do respirador e a utilização de vasodilatadores, tanto pela via venosa como pela via inalatória, foi totalmente ineficaz. No segundo dia, a situação ficou insustentável e, como medida heróica, a paciente foi reconduzida ao centro cirúrgico onde, com auxílio de estase venosa, estabeleceu-se uma CIA, no sentido de aliviar a hipertensão pulmonar. Esta manobra foi realizada em tempo muito curto, mas a paciente tornou-se extremamente cianótica com grave insaturação arterial e, em poucos minutos, apresentou dissociação eletromecânica cardíaca irreversível.

\section{COMENTÁRIOS}

\section{Caso 1. CIA e complicações digestivas após cirurgia} cardíaca

Complicações abdominais da CEC são relativamente raras, correspondendo a menos de $1 \%$ de todos os casos com declínio de sua incidência nas últimas décadas [5]. Por outro lado, a incidência destas complicações chega a ser seis vezes maior de acordo com a equivalência da gravidade nos casos de cirurgia cardíaca [6]. Entre as complicações digestivas mais freqüentes encontram-se a esofagite aguda, as hemorragias digestivas, a icterícia pós-operatória, o abdome agudo inflamatório e a obstrução intestinal. A isquemia e infarto intestinais são menos freqüentes e associam-se com a insuficiência aórtica, doença aterosclerótica e idade entre 40 e 50 anos [7]. O caso apresentado neste texto é único na literatura associando a isquemia mesentérica com a cirurgia corretiva da CIA.

No caso relatado, as possíveis causas de isquemia intestinal e necrose são motivo de especulação. É muito difícil, de um modo geral, relacionar uma causa primária a alguma ocorrência em uma cirurgia de curta duração. No terceiro dia após a cirurgia, a paciente apresentou um episódio súbito de hipotensão, taquicardia e vasodilatação periférica, episódio este de causa desconhecida e de difícil controle. Permaneceu a dúvida se os eventos subseqüentes foram a causa ou conseqüência da isquemia intestinal. Estas dúvidas são comuns a todos os casos semelhantes descritos na literatura. A grande maioria dos casos relatados foi submetida a laparotomia exploradora com extensas ressecções intestinais. Outras medidas incluíram a suspensão do uso de digitálicos e vasoconstritores, bloqueio esplâncnico direto ou epidural, além do uso de $\alpha$ bloqueadores como a fenoxibenzamina. Em todos os casos, apesar do tratamento, a mortalidade foi próxima de $100 \%$, parcialmente associada ao retardo do diagnóstico, uma vez que a maioria dos pacientes apresentava movimentos e ausculta intestinais, tendo a dor como único sintoma. Esta situação incomum ocorreu no paciente descrito: diagnóstico tardio e estado septicêmico.

Permanece como incógnita o fato da incidência de infarto mesentérico não-oclusivo ser incomum após cirurgias com $\mathrm{CEC}$, em pacientes que apresentam débito cardíaco muito baixo no pós-operatório imediato, especialmente entre aqueles que fazem uso do balão intra-aórtico e altas doses de catecolaminas. Este fato leva à especulação sobre uma possível susceptibilidade individual e uma possível alta especificidade do mecanismo fisiopatológico deste raro e catastrófico evento.

A melhor conduta terapêutica ainda é bastante discutível. Embora a quase totalidade dos pacientes descrita na literatura tenha sido submetida a laparotomia exploradora, pelo menos dois relatos mencionam o sucesso do tratamento conservador da isquemia intestinal não-oclusiva através da vasodilatação local $[8,9]$.

Caso 2. CIA e malformações associadas do sistema nervoso central

O número de pacientes portadores de cardiopatias congênitas, que atingem a adolescência e a idade adulta, tem apresentado um aumento contínuo. Uma experiência da Mayo Clinic procurou estudar a incidência de aneurismas intracranianos não-infecciosos e dissecções cérvicocefálicas em pacientes portadores de cardiopatias congênitas, expandindo o espectro das doenças cerebrovasculares que podem ser encontradas neste grupo de pacientes. Este estudo concluiu que as cardiopatias congênitas implicam em maior risco de desenvolvimento de aneurismas intracranianos e dissecções arteriais cérvicocefálicas, particularmente na adolescência. As artérias musculares do crânio e do pescoço são derivadas de células da crista neural, e esta crista é, também, da maior importância no desenvolvimento precoce do coração. A existência dessa estrutura embriológica comum pode ser relevante para a 
associação entre cardiopatias congênitas e malformações arteriais do pescoço e intracranianas [10]. É importante notar que a série da Mayo Clinic, reconhecida mundialmente pela qualidade da sua documentação, não menciona nenhum caso de CIA entre os 14 pacientes portadores de cardiopatia congênita. Esse dado associado ao fato do paciente apresentado ser único na experiência de grupo, com cerca de 7000 cirurgias cardíacas, caracteriza a extrema raridade do evento CIA em associação com aneurisma intracraniano. Uma revisão bibliográfica, tão ampla quanto possível, revelou o relato de apenas um paciente, descrito na Índia, que apresentava associação entre CIA e aneurisma intracraniano [11].

No caso relatado, o diagnóstico foi feito pela avaliação clínica neurológica, punção liquórica e pela realização de uma carotidoangiografia realizada à beira do leito. Por ocasião do evento, ocorrido na década de 80 , não havia condições e nem a rotina de estudos diagnósticos mais sofisticados. Como, a paciente desde o evento hipertensivo acompanhado da ruptura do aneurisma, apresentava-se em quadro sugestivo de morte cerebral não se prosseguiu com a investigação por imagem e nem se indicou uma craniotomia.

\section{Caso 3. CIA e drenagem da veia cava inferior para 0} átrio esquerdo

A drenagem anômala da veia cava inferior (VCI) no átrio esquerdo durante a correção da CIA é uma complicação rara. Uma busca bibliográfica no banco de dados MEDLINE revela um número não superior a 50 casos, a partir de 1966. A anomalia é devida à falha do desenvolvimento embrionário da valva do seio venoso direito, septum secundum e porção dorsal do septo interatrial[12].

Ressalte-se que uma valva de Eustáquio proeminente pode ser confundida com o septo interatrial e ser fechada cirurgicamente $[13,14]$. É preciso enfatizar que a drenagem anômala da VCI em átrio esquerdo pode associar-se a outras cardiopatias congênitas que não a CIA. Embora esta associação seja muito rara, já foram descritas associações com a tetralogia de Fallot [15] e na CIA associada à drenagem anômala de veias pulmonares [16].

O diagnóstico pré-operatório, quando realizado, implica no estudo angiocardiográfico e ecodopplercardiográfico. Usando sofisticações da tecnologia associada à ecocardiografia, tais como a ecocardiografia de contraste ou, ainda, a ecocardiografia tridimensional, este diagnóstico pode ser extremamente refinado [17]. A ressonância magnética é outro recurso de imagem que pode ser utilizado para o diagnóstico da drenagem anômala da veia cava inferior em átrio esquerdo. $\mathrm{O}$ diagnóstico pré-operatório é bastante infreqüente, uma vez que existe algum consenso de que a CIA-II possa ser operada embasada no diagnóstico feito apenas pela ecodopplercardiografia convencional, mas a literatura mostra poucos casos em que o cirurgião realizou a correção cirúrgica já conhecendo a anomalia venosa. Esta suspeição diagnóstica tem como base a associação de cianose com pressão pulmonar normal em recém-natos. $\mathrm{Na}$ maior série, já mencionada acima, o grupo de cardiologistas, seguramente, dirigia a atenção para este tipo de malformação congênita, uma vez que os casos apresentados foram diagnosticados antes do tratamento cirúrgico [18].

$\mathrm{O}$ caso da criança relatado apresenta características compatíveis com a revisão apresentada como parte desta discussão. A indicação cirúrgica foi embasada no quadro clínico, idade pré-escolar e diagnóstico realizado apenas com auxílio da ecodopplercardiografia. $\mathrm{O}$ fato de, no estabelecimento do circuito de CEC, ter sido possível a canulação inferior implica na existência de valva de Eustáquio redundante ou na possibilidade de ter sido canulado algum ramo supra-hepático que drenava diretamente em átrio direito, independente da drenagem anômala da veia cava inferior no átrio esquerdo. É possível, ainda, a reflexão de que a conexão da veia cava inferior no átrio esquerdo possa ser de origem iatrogênica. A persistência da cianose e certo grau de edema de face levaram ao cateterismo cardíaco que revelou a presença da veia cava inferior conectada no átrio esquerdo. A criança foi reoperada, sendo o fluxo da VCI dirigido para o átrio direito utilizando retalhos de pericárdio bovino. Após esta operação, desapareceram a cianose e o edema de face, a criança voltou a tornar-se hiperativa, mas, infelizmente, teve morte súbita em dissociação eletromecânica cardíaca por provável tromboembolismo pulmonar ou, mais provável, por tromboembolismo cerebral. O tromboembolismo pulmonar como causa de morte súbita no pós-operatório recente foi demonstrado em uma criança, cuja autópsia revelou depósitos trombóticos na linha de sutura da CIA-II [19].

\section{Caso 4. CIA e tromboembolismo}

\section{Tromboembolismo cerebral}

O tromboembolismo paradoxal pode ocorrer para qualquer parte do corpo, sendo particularmente grave quando ocorre para o cérebro ou pulmões. $\mathrm{O}$ forame oval patente (FOP), um remanescente embriológico encontrado em $27 \%$ dos adultos, é um potencial sítio de "shunt" intracardíaco direita-esquerda. Este "shunt" pode ser o resultado de uma reversão no gradiente pressórico ou aporte sangüíneo anormal no átrio direito. As conseqüências patológicas do "shunt" direitaesquerda incluem a hipoxemia e o embolismo paradoxal. $\mathrm{O}$ embolismo paradoxal através de um FOP é bem documentado e o seu papel na etiologia do tromboembolismo de origem obscura (tromboembolismo criptogênico) é motivo de grandes discussões e controvérsias [20].

\section{Tromboembolismo pulmonar}


A embolia pulmonar, embora pareça menos freqüente ou menos relatada do que a embolia sistêmica, também pode ser uma intercorrência catastrófica associada à CIA, tanto em casos não operados, muitas vezes ainda sem o diagnóstico de CIA, como após a operação corretiva. A tomografia computadorizada helicoidal e a angioressonância, além da arteriografia pulmonar, são excelentes métodos para o diagnóstico por imagem.

É possível, também, a ocorrência de embolia pulmonar isolada em pacientes com CIA. Esta possibilidade tem sido mais relatada em pacientes idosos. Não existem dados precisos sobre a prevalência da hipertensão pulmonar devida ao tromboembolismo pulmonar. $\mathrm{Na}$ experiência da Universidade de San Diego, a maior casuística de tromboendarterectomia pulmonar, constatou-se ocorrência de até $25 \%$ de CIA ou FOP nos casos operados [21].

As opções para o tratamento cirúrgico são a tromboendarterectomia pulmonar e o transplante pulmonar ou cardiopulmonar. Existem relatos de melhora acentuada da função cardíaca direita com a realização de transplante pulmonar unilateral.

A paciente relatada neste texto havia sido operada há cerca de seis anos para a correção de uma CIA por meio de sutura direta. Sempre manteve queixa, extremamente vaga e incompatível com a sua atividade física. Este sintoma foi valorizado apenas quando a paciente apresentou uma saturação de $88 \%$, detectada em consultório por uma oximetria de pulso. Este dado inesperado desencadeou toda a investigação, a qual revelou grave hipertensão pulmonar com grave comprometimento de cavidades cardíacas direitas e tromboembolismo crônico da circulação pulmonar. Os raciocínios retrospectivos foram todos fundamentados em especulação. O único fator de risco, além da CIA, era a utilização de anticoncepcionais pela paciente. Uma coisa ficou bastante clara, a subestimação de um valor entre $90-100 \mathrm{mmHg}$ antes da correção da CIA, valor este muito elevado para uma CIA de 2,2 cm. Assim, destacam-se três conjeturas: a) Teria esta paciente sido vítima de episódios não detectados de embolias pulmonares pequenas, ou seria portadora de doença endotelial da circulação pulmonar? De qualquer forma, dois ou três anos após a cirurgia, as pressões estimadas pelo ecocardiograma apresentaram valores em torno de $50 \mathrm{mmHg}$. A pesquisa de algum defeito congênito da coagulação foi negativa; b) Teria sido essa paciente vítima de tromboembolismo pulmonar a partir da linha de sutura do septo interatrial? Esta hipótese é improvável, uma vez que o pós-operatório imediato cursou sem intercorrências e; c) Novos episódios tromboembólicos que se tornaram mais freqüentes ou maiores após o terceiro ano da correção da CIA, uma vez que nesta época a pressão estimada da artéria pulmonar estava em torno de $50 \mathrm{mmHg}$ contra $90-100 \mathrm{mmHg}$, no pré-operatório, e $103 \mathrm{mmHg}$, por ocasião do evento final.
Ressalte-se que foi encontrada uma pequena CIA, que não parecia residual e situava-se bem próxima da emergência da veia cava inferior no átrio direito. Este defeito residual poderia ser uma fonte de embolia paradoxal para o pulmão.

\section{Considerações finais}

A principal motivação desse texto está implícita em seu título. As complicações letais da CIA e sua correção cirúrgica são raras e muito pouco relatadas. Será que existe um fator importante que interfere nesta assertiva? Será que os casos são pouco relatados e contribuem para isso o fato do tratamento cirúrgico da CIA ser considerado "uma tarefa para residentes"? De qualquer forma, fica o alerta já mencionado: "não subestime mesmo as mais simples alterações em cirurgia cardíaca". Os quatro casos são fortes exemplos desta assertiva. Como causas dos eventos catastróficos que levaram ao óbito foram destacados: a) eventos isquêmicos gastrointestinais (Caso 1); b) associação da CIA com malformações vasculares do sistema nervoso central (Caso 2); c) problemas associados à drenagem venosa sistêmica anômala (em especial a drenagem da veia cava inferior no átrio esquerdo (Caso 3) e; d) tromboembolismo cerebral (Caso 3?) e pulmonar (Caso 3 e 4?). Uma pergunta que fica no ar diz respeito à prevenção de tais eventos fatais. A resposta é difícil, pois a metodologia do tratamento cirúrgico CIA com circulação extracorpórea é muito bem estabelecida. Se não houver negligência de detalhes, complicações como as apresentadas ficam por conta de trágicas fatalidades.

\section{REFERÊNCIAS BIBLIOGRÁFICAS}

1. Mahoney LT, Truesdell SC, Krzmarzick TR, Lauer RM. Atria septal defects that present in infancy. Am J Dis Child 1986;140:1115-8.

2. Stansel HC, Talner NS, Deren MM, Van Hecekeren D, Glenn WW. Surgical treatment of atrial septal defect: analysis of 150 corrective operations. Am J Surg 1971;121:485-9.

3. Wright J, Albrecht H, Nicholson-Florence M, Newman D, Kearney M, Beveridge J. The optimal age for repair of secundum atrial septal defect. Med J Aust 1983;2:10-1.

4. Gunay I. Do not underestimate atrial septal defect! J Cardiovasc Surg (Torino) 2001;42:773-6

5. Long III WB. Abdominal complications of cardiopulmonary bypass. In: Utley JR, editor. Pathophysiology of cardiopulmonary bypass. Baltimore:Williams and Wilkins;1983. 
6. Harjola PT, Siltanen P, Appelqvist P, Laustela E. Abdominal complications after open heart surgery. Ann Chir Gynaecol Fenn 1968;57:272-4.

7. Hoffman FG, Zimmerman SL, Cardwell Jr. ES. Massive intestinal infarction without vascular occlusion associated with aortic insufficiency. N Engl J Med 1960;263:436-40.

8. Niederhauser U, Genoni M, von Segesser LK, Bruhlmann W, Turina MI. Mesenteric ischemia after a cardiac operation: conservative treatment with local vasodilation. Ann Thorac Surg 1996;61:1817-9.

9. Biraima AM, Genoni M, Winkler MB, Bruhlmann W, Turina MI. Mesenteric ischemia after a cardiac operation: arteriosclerotic versus vasospastic etiology. J Cardiovasc Surg (Torino) 2002;43:87-9.

10. Schievink WI, Mokri B, Piepgras DG, Gittenberger-de Groot AC. Intracranial aneurysms and cervicocephalic arterial dissections associated with congenital heart disease. Neurosurgery 1996;39:685-90.

11. Ramakrishnamurthy TV, Purohit AK, Sundaram C, Ramamurti, Rajender Y. Distal calcarine fusiform aneurysm: a case report and review of literature. Neurol India 1999;47:318-20.

12. Laks H, Ahmad N, Mudd JG. Severe cyanosis due to atrial septal defect and an anomalous inferior vena caval valve. Angiology 1978;29:422-8.

13. Becker A, Buss M, Sebening W, Meisner H, Dohlemann C. Acute inferior cardiac inflow obstruction resulting from inadvertent surgical closure of a prominent Eustachian valve mistaken for an atrial septal defect. Pediatr Cardiol 1999;20:155-7.
14. Maeno YV, Boutin C, Benson LN, Nykanen D, Smallhorn JF. Three-dimensional transesophageal echocardiography for secundum atrial septal defects with a large eustachian valve. Circulation 1999;99:E11.

15. Temkine J, Aigueperse J, Chaumont P, Meyzer JP, Zuber C. Veinous return from the inferior vena cava into the left atrium after surgical correction of Fallot's tetralogy. Arch Mal Coeur Vaiss 1977;70:663-8.

16. Roguin N, Milo S, Isserles S. Atrial septal defect associated with a remnant of the valve of the sinus venosus producing unusual drainage of the inferior caval vein. Int J Cardiol $1986 ; 13: 369-72$

17. Mortera C, Leon G, Martin-Duran R, Sanchez A. Contrast echocardiography in the diagnosis of the surgical diversion of the inferior vena cave into the left ventricle. Rev Esp Cardiol 1981;34:329-32.

18. Hallali P, Tcheng P, Davido A, Leriche H, Corone P. Atrial septal defect and cyanosis. Apropos of 6 cases related to abnormal drainage of the inferior vena cava into the left atrium. Arch Mal Coeur Vaiss 1988; 81:783-6.

19. Busch T, Lotfi S, Sirbu H, Aleksic I, Ruschewski W. Fatal pulmonary embolism after atrial septal defect closure in a paediatric patient. Scand Cardiovasc J 1999;33:187-8.

20. Movsowitz C, Podolsky LA, Meyerowitz CB, Jacobs LE, Kotler MN. Patent foramen ovale: a nonfunctional embryological remnant or a potential cause of significant pathology? J Am Soc Echocardiogr 1992;5:259-70.

21. Jamieson SW, Auger WR, Fedullo PF, Channick RN, Kriett JM, Tarazi RY et al. Experience and results with 150 pulmonary thromboendarterectomy operations over 29-month period. J Thorac Cardiovasc Surg 1993;106:116-27. 